\title{
The dynamical state and blue straggler population of the globular cluster NGC 6266 (M62) ${ }^{1}$
}

\author{
G. Beccari ${ }^{1,2,3}$, F.R. Ferraro ${ }^{4}$, A. Possenti ${ }^{5}$, E. Valenti ${ }^{4,1}$, L. Origlia ${ }^{1}$, R.T. Rood ${ }^{6}$ \\ ${ }^{1}$ INAF-Osservatorio Astronomico di Bologna, via Ranzani 1, I-40127 Bologna, Italy, \\ giacomo.beccari bo.astro.it \\ ${ }^{2}$ Dipartimento di Scienze della Comunicazione, Università degli Studi di Teramo, Italy \\ ${ }^{3}$ INAF-Osservatorio Astronomico di Collurania, Via Mentore Maggini, I-64100 Teramo, \\ Italy \\ ${ }^{4}$ Dipartimento di Astronomia, Università degli Studi di Bologna, via Ranzani 1, I-40127 \\ Bologna, Italy, francesco.ferraro3 unibo.it \\ ${ }^{5}$ INAF-Osservatorio Astronomico di Cagliari, Loc. Poggio dei Pini, Strada 54, I-09012 \\ Capoterra, Italy, possenti ca.astro.it \\ ${ }^{6}$ Astronomy Department, University of Virginia, Charlottesville, VA, 22903, \\ rtr virginia.edu
}

\begin{abstract}
We have used a proper combination of multiband high-resolution HSTWFPC2 and wide-field ground based observations to image the galactic globular cluster NGC 6266 (M62). The extensive photometric data set allows us to determine the center of gravity and to construct the most extended radial profile ever published for this cluster including, for the first time, detailed star counts in the very inner region. The star density profile is well reproduced by a standard King model with an extended core $\left(\sim 19^{\prime \prime}\right)$ and a modest value of the concentration parameter $(c=1.5)$, indicating that the cluster has not-yet experienced core collapse.

The millisecond pulsar population (whose members are all in binary systems) and the X-ray emitting population (more than 50 sources within the cluster half mass radius) suggest that NGC 6266 is in a dynamical phase particularly active in generating binaries through dynamical encounters. UV observations of the central region have been used to probe the population of blue straggler stars, whose origin might be also affected by dynamical interactions. The comparison with other globular clusters observed with a similar strategy shows that the blue
\end{abstract}


straggler content in NGC 6266 is relatively low, suggesting that the formation channel that produces binary systems hosting neutron stars or white dwarfs is not effective in significantly increasing the blue straggler population. Moreover, an anticorrelation between millisecond pulsar content and blue straggler specific frequency in globular cluster seems emerging with increasing evidence.

Subject headings: Globular clusters: individual (NGC 6266); stars: evolution binaries: close - blue stragglers

\section{Introduction}

In the last decades it has become obvious that some stars observed in Globular Clusters (GCs) are not the products of the passive evolution of isolated stars. Stellar encounters can produce binary systems and affect the evolution of the new produced binaries as well as any binaries that might have existed since the formation of the cluster. Thus the stellar content of a GC is intimately linked with the dynamical history of the cluster. In fact, the Color-Magnitude Diagrams (CMDs) of the core regions of GCs recently observed using HST-WFPC2 have shown the presence of a variety of exotic stellar objects, whose formation and evolution may be strongly affected by dynamical interactions. These include Low-Mass X-ray Binaries (LMXBs), (e.g. Heinke et al. 2001, Edmonds et al. 2003), Cataclysmic Variables (CVs) (e.g. Cool et al. 1998), and Blue Straggler Stars (BSSs) (e.g. the cases of 47 Tuc, Ferraro et al 2004; M3, Ferraro et al. 1997; M80, Ferraro et al., 1999a; M30, Guhathakurta et al., 1998, Ferraro et al. 2003a, hereafter F03).

BSS were first discovered by Sandage (1953) in the globular cluster M3, and manifest themselves in the CMD as an extension of the Main Sequence (MS) above the Turn-Off (TO), mimicking MS stars with larger initial masses. There are two viable proposed mechanisms for BSS generation: the first is mass exchange in a primordial binary system and the second is the merger of two stars induced by stellar interactions (either single or binary) in a dense stellar environment. The fact that BSSs have been found in all properly observed stellar systems make them a powerful tracer of dynamical history of the parent cluster.

\footnotetext{
${ }^{1}$ Based on observations with the NASA/ESA HST, obtained at the Space Telescope Science Institute, which is operated by AURA, Inc., under NASA contract NAS5-26555. Also based on WFI observations collected at the European Southern Observatory (ESO), La Silla, Chile, within the observing programmes 62.L-0354 and 64.L-0439.
} 
Millisecond Pulsars (MSPs) may also be the by-product of collisions in the dense environment of a cluster. They form in binaries containing a neutron star (NS) which is eventually spun up through mass accretion from the evolving companion. Since they are point-like objects and (in many cases) extremely stable clocks, they are invaluable tools to investigate the binary evolution in a very dense stellar environment (e.g. Rappaport et al. 2001). It is important to study the overall properties of GCs hosting MSPs and to compare the distributions of the objects which may be significantly affected by dynamical encounters. Hence we have undertaken a long-term project to observe in the optical band the GCs which are rich in known MSPs, with particular emphasis on the BSS population. After having examined 47 Tucanae (Ferraro et al. 2001, 2004; Mapelli et al. 2004) and NGC 6752 (Ferraro et al. 2003b, Sabbi et al. 2004), we now turn our attention to NGC 6266.

NGC $6266(\mathrm{M} 62)$ is a high density $\left(\log \rho_{c} \sim 5.34\right)$, highly reddened $(E(B-V) \sim 0.47)$ GC (Jacoby 2002; Possenti et al. 2003, hereafter P03) and is one of the most massive $\left(M_{v}=-9.19\right.$, Harris $\left.1996^{2}\right)$. It has been classified as a possible Post Core Collapse (PCC) GC in the compilation by Djorgovski \& Meylan (1993, hereafter DM93). Six binary MSPs have been recently discovered in this cluster (D'Amico et al. 2001a, 2001b; Jacoby et al. 2002; P03). NGC 6266 ranks fourth of the GCs in wealth of MSPs, after Terzan 5, 47 Tucanae and M15. Surprisingly, all MSPs in NGC 6266 are in binary systems (P03). As discussed in P03, the absence of known isolated MSPs in NGC 6266 cannot simply be ascribed to a selection effect since, for a given spin period and flux density, an isolated MSP is easier to detect than a binary MSP. If such a lack of isolated pulsars is not a statistical fluctuation, it must be somehow related to their formation mechanism and to the dynamical state of the cluster. Moreover, recent deep Chandra X-ray observations of NGC 6266 have revealed a very large number of X-ray sources - 51 sources were detected within the cluster 1'.23 half mass radius (Pooley et al. 2003), indicating that an high number of cataclysmic (and/or interacting) binaries should be present. These observational facts may indicate that NGC 6266 is now in a dynamical state where the rate $\mathcal{R}_{\text {form }}$ of formation (and of hardening) of binary systems containing a neutron star and/or a white dwarf is much larger than the rate of disruption $\mathcal{R}_{\text {disr }}$ of such systems.

In order to investigate in more detail the dynamical properties of this cluster, we have used HST-WFPC2 high resolution images of the central core and ESO-WFI wide field observations to sample the more external regions. The combination of these two data-sets allows us to derive an accurate star density profile over the entire cluster extension. $\S 2$ describes the datasets and the reduction procedures, while $\S 3$ presents the derived CMD. $\S 4$ describes

\footnotetext{
${ }^{2}$ For all references to Harris (1996) we have used the updated data set at the web site http://www.physics.mcmaster.ca/Globular.html
} 
the determination of the cluster center of gravity and the star density profile whereas $\S 5$ is focused on the determination of the new cluster parameters. $\S 6$ is devoted to the study of the Blue Straggler population in the central regions of NGC 6266 and, finally we discuss the results in $\S 7$.

\section{Observations and data analysis}

The photometric data used here consist of two sets. (i) - The high spatial resolution set includes a series of HST-WFPC2 images obtained in August 2000, through the F555W $(V)$, F336W $(U)$, and F255W $(U V)$ filters as part of a long term project (GO-8709, PI: F. R. Ferraro) aimed at studying the central stellar populations in a sample of GCs. In this dataset the planetary camera (PC, with the highest resolution of $\sim 0^{\prime \prime} .046 /$ pixel) is roughly centered on the cluster center, while the WF cameras (at a lower resolution of $\sim 0$ ' $1 /$ pixel) sample the surrounding outer regions. (ii) - The wide field set-secured at the $2.2 \mathrm{~m}$ ESOMPI telescope at ESO (La Silla) in July 2000 using the WFI, which has exceptional imaging capabilities by providing a mosaic of 8 CCD chips (each with a field of view of $8^{\prime} \times 16^{\prime}$ ) for a global field of view of $33^{\prime} \times 34^{\prime}$. The cluster is roughly centered on chip \#2 and observed through the $B, V, I$ broad band filters.

\subsection{Photometry and Astrometry}

The raw ground-based WFI images were corrected for bias and flat field, by using standard $\mathrm{IRAF}^{3}$ tools. The point spread function (PSF) fitting procedure was performed independently on each $B, V$, and $I$ image, using DAOPHOT II (Stetson 1994). A final catalog listing the instrumental $B, V$, and $I$ magnitudes for all the stars in each field has been obtained by cross-correlating the three catalogs. The WFI catalog was finally calibrated by using the data-set by Rosenberg et al. (2000) for I magnitudes and by Brocato et al. (1996) for $V$ and $B$ magnitudes.

The photometric reduction of the high resolution HST images was carried out using ROMAFOT (Buonanno et al. 1983), a package developed to perform accurate photometry in crowded fields and specifically optimized to handle under-sampled PSFs (Buonanno \&

\footnotetext{
${ }^{3}$ IRAF is distributed by the National Optical Astronomy Observatory, which is operated by the Association of Universities for Research in Astronomy, Inc., under cooperative agreement with the National Science Foundation.
} 
Iannicola 1989), as in the case of the HST-WF cameras. PSF-fitting instrumental magnitudes have been obtained using the standard procedure described in Ferraro et al. (1997, 2001). The final catalog of the F336W, F555W and F255W magnitudes was calibrated by using the zero-points listed by Holtzman et al. (1995). The HST F555W band photometry has been then transformed into the Johnson $V$ system by using the stars in common between the WFPC2 and WFI catalogs. The photometric error of the final catalogs is dominated by the zero-point calibration uncertainties of $\approx \pm 0.05$ mag.

The Guide Star Catalog (GSCII) was used to search for astrometric standards in the entire WFI image field of view. Several hundred astrometric GSCII reference stars were found in each chip, allowing an accurate absolute positioning of the sources. An astrometric solution has been obtained for each of the 8 WFI chips independently, by using suitable catalog matching and cross-correlation tools developed at the Bologna Observatory . At the end of the entire procedure, the rms residuals are $\approx 0^{\prime \prime} 2$ both in RA and Dec.

The small field of view (2'.5 on the side) of the high resolution HST-WFPC2 images is entirely contained within the WFI chip \#2, which imaged the cluster core. We used more than 2500 bright stars in the WFI catalog lying in the WFPC2 field of view as secondary astrometric standards, in order to properly find an astrometric solution for this catalog as well. We estimate that the global uncertainty in the astrometric solution is $\leq 0^{\prime \prime} .3$ both in RA and Dec.

A master, homogeneous catalog of magnitudes and absolute coordinates including all the stars in the HST and the WFI catalog was finally produced.

\section{The CMD of NGC 6266}

Figure 1 show the CMD in the $(V, U-V)$ plane for the entire HST sample. As can be seen all the cluster sequences are clearly defined and well populated. Particularly notable is the Horizontal Branch (HB) morphology. The CMD clearly shows an extended HB tail: this feature was already suspected on the basis of previous photometry (see for example Caloi et al. 1987, Brocato et al. 1996, and Contreras et al. 2005) but its true extension is revealed for the first time by the diagram shown in Figure 1 (see also Piotto et al., 2002). The HB tail extends $\sim 1$ mag below the MSTO and it is not uniformly populated. Similar morphology has been already observed in other clusters (NGC 2808 - Sosin et al 1997; M13-Ferraro et al . 1997; M80-Ferraro et al. 1998; NGC 6752-Ferraro et al. 2003b, see also Catelan et al. 1998, Piotto et al. 1999 and Ferraro et al 1998 for a discussion on the reality of gaps along the HB). We defer the detailed discussion of the stellar distribution along the HB to 
a future paper, where the HB morphology of these clusters will be compared to each other.

NGC 6266 is in the sample of 62 GCs for which Ferraro et al. (1999b, hereafter F99) derived distance modulus and photometric properties. In doing this, F99 used the photometry presented by Brocato et al (1996). As can be seen from their Figure 12b the HB appears quite dispersed when compared with the CMD shown here in Figure 1. Hence, since the zero point of the photometry presented here is based on the photometry by Brocato et al (1996), we used our new photometry in order to derive a more accurate level of the ZAHB $\left(V_{Z A H B}\right)$. Following the procedure described in F99, we obtained $V_{Z A H B}=16.25 \pm 0.10$, significantly $(\delta V=0.15)$ brighter than the values in F99. By adopting this new value, the reddening from Harris (1996) $(E(B-V)=0.47)$ and the metallicity from Carretta\& Gratton (1997, hereafter CG97) $\left([\mathrm{Fe} / \mathrm{H}]_{\mathrm{CG} 97}=-1.07\right)$, we derived (from equation 4 in F99) $M_{V}^{Z A H B}=0.68$ , an apparent distance modulus of $(m-M)_{V}=15.57 \pm 0.15$ and a true distance modulus of $(m-M)_{0}=14.11 \pm 0.15$ which corresponds to a distance of $6.6 \mathrm{Kpc}$.

Recently Contreras et al. 2005 have found a large (> 200) population of RR Lyrae in NGC 6266. In order to determine the number of RR Lyrae variables lying in the field sampled by the HST-WFPC2, we cross-correlated our catalog with the list of variable stars by Contreras et al (2005). We identified 52 RR Lyrae in the HST/WFPC2 field of view. The positions of these stars in the CMD of Figure 1 are marked by large filled triangles. We also identified 68 RR Lyrae in the WFI catalogue. The mean magnitude of all 126 identified variables is $\left\langle V_{\mathrm{RRLy}}\right\rangle=16.16 \pm 0.4$. By adopting the relation:

$$
M_{V}(\mathrm{HB})=0.22([\mathrm{Fe} / \mathrm{H}]+1.5)+0.56
$$

by Gratton et al (2003) and the metallicity of NGC $6266\left([\mathrm{Fe} / \mathrm{H}]_{\mathrm{CG} 97}=-1.07\right)$ we found $M_{V}^{\mathrm{HB}}=0.65$ and an apparent distance modulus $(m-M)_{V}=15.55 \pm 0.15$, which is fully consistent with the value reported above. Hence in the following we adopt $(m-M)_{V}=$ $15.57 \pm 0.15, E(B-V)=0.47$ and $(m-M)_{0}=14.11 \pm 0.15$.

\section{The surface brightness and star density profile}

\subsection{The center of gravity of NGC 6266}

For the first time star counts in the central region of the cluster can be computed using the CMD presented in Figure 1. Ferraro et al. (2003b, 2004) have shown that the knowledge of the position of individual stars in the innermost region of the cluster allows a high precision determination of the position of the center of gravity. To do this we applied the procedure described in Montegriffo et al. (1995) (see also Calzetti et al. 1993) and we computed 
$C_{\text {grav }}$ by simply averaging the $\alpha$ and $\delta$ coordinates of stars lying in the PC camera of the HST catalog. In order to evaluate any possible spurious effect due to incompleteness of the sample in the very inner region of the cluster, we considered samples with different limiting magnitudes $\left(m_{\mathrm{F} 555 \mathrm{~W}}<20.5,20\right.$ respectively). For each sample we computed the barycenter of the stars by using an iterative procedure (Ferraro et al 2003b). Both the determinations agreed within less than $\sim 1^{\prime \prime}$. The center of gravity of the cluster $\left(C_{\text {grav }}\right)$ turns out to be located at $\alpha_{\mathrm{J} 2000}=17^{\mathrm{h}} 01^{\mathrm{m}} 12^{\mathrm{s}} .78, \delta_{J 2000}=-30^{\circ} 06^{\prime} 46^{\prime \prime} .0$ with a typical $1 \sigma$ uncertainty of $0^{\prime \prime} .5$ in both $\alpha_{\mathrm{J} 2000}$ and $\delta_{\mathrm{J} 2000}$, corresponding to about 10 pixels in the PC image.

Figure 2 shows the $10^{\prime \prime} \times 10^{\prime \prime}$ computer map around the cluster $C_{\text {grav }}$ as determined in this work (marked with a large cross at $(0,0)$ ). The cluster luminosity center derived by DM93 (small cross in Figure 2) is at about $3^{\prime \prime}$ NE from our determination, whereas the center of Harris (1996)is about $3^{\prime \prime}$ south.

\subsection{The star density and surface brightness profile}

The extended data set collected for this cluster offered the possibility to compute the radial star density profile. As first step, we define the star sample, paying particular care to avoid spurious effects due to possible incompleteness. Since such effects could be particularly important in the WFI sample, we restricted it to stars with $r>140^{\prime \prime}$. In the HST sample we have excluded the external region of the sample since the particular geometry of the WFPC2 field of view prevents an appropriate sampling of concentric annuli used to compute the radial density profile. A limiting magnitude of $V \sim 20.5$ was adopted for both samples. To summarize:

1. the WFPC2/HST sample: all stars detected in the WFPC2 catalog with $V<20.5$ and $r<93^{\prime \prime}$ from the cluster center;

2. the WFI sample: all stars detected in the WFI catalog with $V<20.5$ and $r>140^{\prime \prime}$ from the cluster center.

Figure 3 shows the CMD in the $(V, U-V)$ and $(V, B-V)$ planes for the HST and WFI samples, respectively. As can be seen, the CMD of the WFI catalog (right panel in Figure 3) is severely contaminated by the disk population, which defines the almost vertical blue sequence (at $0.5<B-V<1.2$ ) and, the bulge population at $(B-V) \simeq 1.5$, which clearly indicates the presence of metal-rich stars. Figure 3 shows also a bright portion of the cluster Red Giant Branch (RGB) at $V \sim 14$ and $B-V \sim 1.5$ and, the blue extension of the HB at $V \sim 16$ and $B-V<0.5$. 
By using the combined data set shown in Figure 3 we computed the star density and surface brightness profiles, applying the standard procedure already described in previous papers (see Ferraro et al. 1999a, 2004). The entire photometric sample has been divided in 41 concentric annuli centered on $C_{\text {grav }}$, spanning a spatial range from $0^{\prime \prime}$ to $25^{\prime}$. Each annulus has been split in a number of sub-sectors (generally octants or quadrants, depending on the geometry of the field covered by the HST and WFI fields). The number of stars lying within each sub-sector was counted and star density was obtained by dividing the average star number by the area of the sub-sector. The surface brightness of each sub-sector has been computed by summing the luminosity of all the stars lying inside it, and normalizing the total luminosity to the subsector area.

The average of the star density and surface brightness calculated in each subsectors, at a given radius, yields the stellar density and surface brightness of the parent annulus, respectively. The uncertainty in the average values for each annulus was estimated from the variance among the subsectors. Errors in the number counts can also be estimated from Poison counting statistics and are consistent with the empirically determined values.

The star density values obtained for each annulus at different distance from the cluster center and the estimated errors are listed in Table 1 and shown in Figure 4 . This is the most complete and extended density profile ever published for this cluster, since it samples the cluster population from the very inner core region out to $r \sim 25^{\prime}$ from the cluster center.

The computed surface brightness can suffer relatively large fluctuations due to the small number statistics of the bright giants. Hence, we computed three radial profiles, removing the stars brighter than $V=12,13,14$, respectively. Figure 5 shows that the overall structure of the profile does not change with the adopted magnitude limit. In contrast, the dispersion among different sub-sectors significantly decreases once the brightest stars are excluded.

However, the surface profile shown in Figure 5 indicates a central brightness of $\mu_{v, 0} \sim$ $15.2 \mathrm{mag} \operatorname{arcsec}^{-2}$. This value is fully consistent with that listed by Harris (1996) $\left(\mu_{v, 0} \sim\right.$ $\left.15.35 \mathrm{mag} \operatorname{arcsec}^{-2}\right)$ and DM93 $\left(\mu_{v, 0} \sim 15.19 \mathrm{mag} / \operatorname{arcsec}^{2}\right)$, hence in the following we adopt this value for the central surface brightness of the cluster.

\subsection{The dynamical state of NGC 6266}

The shape of the radial density profile of GCs is generally used to infer the dynamical state of the system (see the pioneering work by King 1966 and Djorgovski \& King 1986). In particular, Djorgovski \& King (1986) defined two classes of clusters: the so-called King Model (KM) cluster and the PCC cluster, depending of the central density profile. KM clusters have 
density profiles which can be reproduced by a single-component King Model (King 1966) with a flat isothermal core and a steep envelope characterized by two parameters: the core radius $\left(r_{c}\right)$ and the tidal radius $\left(r_{t}\right)$ or, alternatively, the concentration $c=\log \left(r_{t} / r_{c}\right)$. The star density profile of a PCC cluster follows an almost pure power law with an exponent $\sim 1$. However Meylan \& Heggie (1997) stated that the general dynamical status of a cluster can be deduced directly from the concentration value "c", since all clusters with high concentration parameter value $(c>2)$ can be considered as collapsed or on the verge of collapsing.

In order to model the observed profile, isotropic, single-mass King-models projected onto the cluster area have been computed by using the Sigurdsson \& Phinney (1995) code. As is shown in Figure 4, the observed radial profile is well reproduced by a KM with an extended core $\left(r_{c}=19^{\prime \prime}\right)$. Note that the value of the core radius is significantly larger (almost by a factor of 2$)$ and the concentration $(c=1.5)$ significantly lower than $r_{c}=10^{\prime \prime} .7$ and $c=1.7$ tabulated by Trager et al. (1995). Since the star density profile is not affected by statistical fluctuations due to the presence of a few bright giants, it offers the best route for deriving cluster parameters (see also Lugger, Cohn \& Grindlay 1985 and Ferraro et al 2003b).

Both the existence of an extended core and a relatively low value of the concentration parameter suggest that the cluster has not yet experienced the collapse of the core. Hence, the first result of this study is that NGC 6266 is not a PCC cluster.

The star density profile shown in Figure 4 also allows us to evaluate the contribution of the background stellar population, which is substantial due to the fact that the cluster is located in the direction of the Galactic Bulge. Our star counts can be used to evaluate the projected Bulge star density down to $V \sim 20.5$. By averaging star counts in the region where the radial profile become flat (corresponding to $r>470^{\prime \prime}$ from the cluster center), we have estimated a value of $\sim 92$ stars $\operatorname{arcmin}^{-2}$. The background level is shown as a dashed

horizontal line in Figure 4. The poorer fit of the King Model to the cluster in the region of transition to the background could arise from our modeling of the background as spatially uniform.

\section{The new cluster parameters}

Since the KM fitting of the observed stellar density profile of the cluster suggested values of $r_{c}$ and $c$ significantly different from those previously measured, we used them to re-determine the cluster structural parameters. In doing this, we adopted the equations given by Djorgovski 1993 (from here D93). Following his assumptions the central luminosity density $\rho_{0}^{L}$ is: 


$$
\rho_{0}^{L}=\Sigma_{0} /\left(r_{c} p\right)
$$

where $r_{c}$ is in parsecs and $\Sigma_{0}$ is the central surface brightness in $L_{V \odot} \mathrm{pc}^{-2}$, evaluated as follows:

$$
\log \left(\Sigma_{0}\right)=0.4\left[26.362-\mu_{V, 0}(0)\right]
$$

and $p$ is a function which depends on the cluster concentration $(c)$, given by the following expression:

$$
\log (p)=-0.603 \times 10^{-c}+0.302
$$

and $\mu_{V, 0}(0)$ is the central brightness of the cluster corrected for extinction.

In computing these quantities we adopted the distance modulus and the reddening discussed in Section 3. The resulting central luminosity density is $\log \left(\rho_{0}^{L}\right)=4.98\left[L_{\odot} \mathrm{pc}^{-3}\right]$. If, according to D93, we use a mass-to-light ratio $\left(M / L_{V}\right)=3$ for converting the central luminosity density into the central mass density, we derive a central density value of $\log \rho_{0}=5.46\left[\left(M_{\odot} \mathrm{pc}^{-3}\right)\right]$, which has to be compared with the value listed in D93 $\log \rho_{0}=5.7\left[\left(M_{\odot} \mathrm{pc}^{-3}\right)\right]$. All the derived parameters are listed in Table 2 along with the values by DM93 for comparison.

To homogeneously compare the properties of NGC 6266 with those of other GCs, we also calculated new values of $\rho_{0}$ for a sample of GCs previously studied with similar techniques (see F03, Sabbi et al. 2004, Ferraro et al. 2004). In doing this we adopted the distance scale defined by F99 (also the adopted reddening is from Table 2 by F99) and the $r_{c}$ derived from the radial density profile of each cluster (see F03, Mapelli et al. 2004, Ferraro et al. 2003b). Results are listed in Table 3 along with the previous determinations by D93. The comparison shows that the different distance modulus adopted here (with respect to D93) has a direct effect on the derived value of the cluster central density, with a major impact on the derived binary-binary collision rate (see Section 7).

\section{The population of Blue Straggler Stars in the core of NGC 6266}

Special classes of stars, in particular those resulting from the evolution of binary systems, can be used as tracers of the GC dynamical evolution. This is the case of the BSS. From an observational point of view, the high angular resolution and UV imaging capabilities of HST 
has allowed searches for BSS candidates in the core of highly concentrated GCs leading to a burst of activity in this sector (see for example Ferraro et al. 1997, 1999a, F03; Piotto et al. 2004).

An obvious difficulty in studying BSS is the definition of an unequivocal selection criterion since the BSS sequence generally merges into the normal cluster MS without any discontinuity. However, previous work done by our group (Ferraro et al. 1997, 1999a, 2001, F03, 2004) has shown that the UV plane (the $m_{255}, m_{255}-m_{336}$ plane in particular) is ideal for selecting BSS. In this plane the BSS sequence appears almost vertical and significantly brighter than the TO and Sub Giant Branch (SGB). In order to properly compare the BSS population in different clusters, here we follow the same procedure defined in Ferraro et al. 1997 and adopted in F03. A limiting magnitude $m_{F 255 W}=19$ has been adopted in selecting bright BSS once the CMD of each cluster has been shifted to match that of M3. Following this criterion, 47 BSS candidates have been selected in NGC 6266. Figure 6 (Left panel) shows the selection box adopted for the BSS in the UV plane. The position of the selected BSS in the $(V, U-V)$-CMD is also shown in the Right panel of Figure 6.

Since BSS are the result of two stars merging in a binary system, one can expect that in a dynamically relaxed stellar system, BSS are centrally concentrated with respect to normal stars (see Bailyn 1995) because of mass segregation. Indeed, the central relaxation time of even a sparse cluster is significantly lower than the lifetime of a 1-1.5 $M_{\odot}$ MS star. Mass segregation has been observed in all the GCs properly observed up to now (see F03 and reference therein) except $\omega$ Centauri (Ferraro et al. 2005), which may not be a true GC (Tsuchiya et al., 2004).

When using UV-CMDs the obvious reference population is the HB, which appears quite luminous and well defined. HB stars have masses $\left(\sim 0.5-0.8 M_{\odot}\right)$ significantly smaller than those of BSS and their lifetimes are typically shorter than GC relaxation times. Figure 6 (Left panel) shows the adopted selection box for HB stars in the UV plane. Indeed they are easely separable from the cooler (hence fainter) RGB stars. The right panel of Figure 6 shows their position in the (V,U-V) CMD. As can be seen, the selection boxes defined in the UV plane assure the proper inclusion of the entire HB populations down to the faintest end of the branch. In the WFPC2 field of view, we have identified a total population of $395 \mathrm{HB}$ stars (52 RRLyrae - see Section 3 and 343 non variable stars, respectively) ${ }^{4}$.

We have determined the cumulative radial distribution of BSS and compared to HB stars as a function of the projected distance from the cluster center. Figure 7 shows the result. It is evident from the plot that BSS (solid line) are significantly more concentrated toward the

\footnotetext{
${ }^{4}$ Note that only non-variable HB stars have been plotted in both panels of Figure 6
} 
center than HB stars. We applied a Kolmogorov-Smirnov test to both distributions to check the statistical significance of the detected difference: the probability that the BSS population and the HB stars are extracted from the same parent distribution is $P=2 \times 10^{-4}$. Thus, the central concentration of the BSS is confirmed with a $>99 \%$ statistical significance.

\subsection{BSS Specific Frequency}

In order to make a direct comparison with the BSS population in the previous published GCs (Ferraro 1997, F03, Sabbi et al 2004, etc), we computed the BSS specific frequency. As an example, Figure 8 shows the UV-CMD of NGC 6266 (left panel) compared to that of M80 (right panel) presented in F03. From Ferraro et al. (1999a), the specific frequency $\left(F_{\mathrm{HB}}^{\mathrm{BSS}}\right)$ is defined as the ratio between the number of BSS $\left(N_{\mathrm{BSS}}\right)$ to the number of HB $\left(N_{\mathrm{HB}}\right)$ stars. It's worth noticing that the number of stars in each post-MS phase is an excellent indicator of the sampled cluster luminosity (see Renzini \& Buzzoni 1986). Hence $F_{\mathrm{HB}}^{\mathrm{BSS}}$ is a suitable tool for a quantitative comparison between BSS populations in different GCs. A further advantage is that $F_{\mathrm{HB}}^{\mathrm{BSS}}$ is a purely observational quantity and can be easily computed in the UV-CMDs, where the HB population is well separated from the other sequences. The values of $F_{\mathrm{HB}}^{\mathrm{BSS}}$ obtained in 6 GCs by F03, in NGC 6752 by Sabbi et al. 2004, in 47 Tucanae by Ferraro et al. 2004 and the one calculated in NGC 6266 are reported in Table 4, sorted according to increasing $F_{\mathrm{HB}}^{\mathrm{BSS}}$ (the values of $N_{\mathrm{BSS}}$ and $N_{\mathrm{HB}}$ are also reported). All the clusters listed in Table 4 have been observed in the central regions by using the WFPC2/HST and hence represent an homogeneous sample for the sake of comparison.

We found a particularly low BSS specific frequency $\left(F_{\mathrm{HB}}^{\mathrm{BSS}}=0.13\right)$ in NGC 6266 , a value that lies between that of M13 and 47Tuc. It is interesting to notice that NGC 6266 is the highest central density cluster in the sample but it has one of the lowest BSS specific frequencies. On the other hand F03 noticed that NGC 288, the cluster with the lowest central density in their sample, shows the largest BSS specific frequency. This is further evidence that the central density of a cluster is not the driving factor in determining the number of BSS (see also Davies et al., 2004).

\section{Discussion and Conclusions}

By adopting the cluster parameters computed in Section 5, we can now investigate some dynamical properties of NGC 6266 . We computed the mean time interval between singlesingle $\left(t_{s s}\right)$ and binary-binary $\left(t_{b b}\right)$ collisions using equations 13 and 14 respectively from 
Leonard (1989):

$$
\begin{aligned}
t_{s s} & =5.5 \times 10^{9}\left(\frac{1 \mathrm{pc}}{r_{c}}\right)^{3}\left(\frac{10^{3} \mathrm{pc}^{-3}}{n_{0}}\right)^{2} \times\left(\frac{V_{r m s}}{5 \mathrm{~km} \mathrm{~s}^{-1}}\right)\left(\frac{0.5 M_{\odot}}{m_{*}}\right)\left(\frac{0.5 R_{\odot}}{R_{*}}\right)[y r], \\
t_{b b} & =1.7 \times 10^{7}\left(\frac{1 \mathrm{pc}}{r_{c}}\right)^{3}\left(\frac{10^{3} \mathrm{pc}^{-3}}{n_{0}}\right)^{2} \times\left(\frac{V_{r m s}}{5 \mathrm{~km} \mathrm{~s}^{-1}}\right)\left(\frac{0.5 M_{\odot}}{m_{*}}\right)\left(\frac{1 A U}{a}\right)[y r],
\end{aligned}
$$

where $a$ is the initial semimajor axis of each binary which can be estimated for each cluster according to the relation (F03)

$$
a=\frac{G M}{9 \sigma_{0}^{2}},
$$

$n_{0}$ is the central number density (number of stars per parsec ${ }^{3}$ ) and $V_{r m s}$ is the relative rootmean-square velocity, which can be approximated with the central velocity dispersion $\left(\sigma_{0}\right.$, see Table 5). Leonard (1989) derived his equation (14) under the assumption that the binary frequency is $100 \%$ in the core and the star mass is $m_{*}=0.5 M_{\odot}$. Assuming an average mass of $m_{*}=0.2 M_{\odot}$ (Kroupa 2001) as suggested in F03, and $R_{*}=0.5 R_{\odot}$, we computed the number of single-single $(s s)$ and binary-binary $(b b)$ encounters occurring per Gyr $\left(N_{s s, b b}\right)$ in the core of NGC 6266 to be:

$$
\left.N_{s s, b b}=\frac{10^{9}}{t_{s s, b b}} \quad \text { [encounters } \mathrm{Gyr}^{-1}\right] .
$$

In Table 5 we give the results obtained for NGC 6266, compared to the 8 GCs which have been previously searched for BSS in the central regions by using the same observational strategy. The values of the encounter rate are normalized to those obtained for 47 Tuc. NGC 6266 has a predicted $b b$ encounter rate $N_{b b}$ which is much greater than for any other analyzed GC. As shown in Table 5, the same result holds for the close encounters between two single stars leading to tidal capture. Both of these classes of dynamical interactions (together with the binary-single $[b s]$ encounters) may lead to the merging of the two stars, thus potentially producing a BSS. Since all collision modes should be effective in increasing the population of BSS, one would expect to see a relatively larger specific frequency of BSS in NGC 6266. This expectation is not born out by the results of Table 4. The discrepancy might be reduced if the binary fraction in NGC 6266 were significantly smaller than in the other GCs, but this hypothesis can hardly be reconciled with the estimated high rate of binary formation $\mathcal{R}_{\text {form }}$ computed for this cluster (see later). Perhaps the results given in Tables 4 and 5 can be understood if the BSS in NGC 6266 result from primordial binaries 
which have survived in the outer part of the cluster eventually wandering into the center where interactions drive them to merge (as we suggested for 47 Tuc, see Ferraro et al. 2004).

On another side, the very high rate of binary-binary and binary-single star interactions in NGC 6266 may well explain why all of its known MSPs are in binary systems. In fact, P03 suggested that NGC 6266 is in a particular dynamical phase in which the rate of binary system formation $\mathcal{R}_{\text {form }}$ is larger than their destruction rate $\left(\mathcal{R}_{\text {disr }}\right)$. Following the prescription of Verbunt (2003), we recomputed those ratios for all clusters listed in Table 6. According to Verbunt $(2003) \mathcal{R}_{\text {form }} \propto \rho_{0}^{1.5} r_{c}^{2}$ and $\mathcal{R}_{\text {disr }} \propto \rho_{0}^{0.5} r_{c}^{-1}$, where $\rho_{0}$ is the central density (expressed in $M_{\odot} \mathrm{pc}^{-3}$ ) and $r_{c}$ is the core radius of the cluster. In Table 6 we give the values of $\mathcal{R}_{\text {form }}$ and $\mathcal{R}_{\text {disr }}$ normalized to 47 Tuc (as done by P03). A quite useful quantity is the ratio $\mathcal{R}_{\text {form }} / \mathcal{R}_{\text {disr }}$ which quantifies the binary survival rate in each environment. NGC 6266 shows, by far, the largest value of this ratio among the clusters listed in Table 6. The 2nd ranked cluster is M3 in which all the MSPs discovered so far are also in binaries.

In summary, we have been able to recompute the ratio $\mathcal{R}_{\text {form }} / \mathcal{R}_{\text {disr }}$ and to show that (i) its high value nicely agrees with the hypothesis (supported by observations of MSPs and X-ray sources) that NGC 6266 has experienced (or is experiencing) a phase of very high production of binary systems. Additionally, we show that (ii) the highly effective binary production phase is occurring while the cluster has not yet undergone the collapse of the core, and that (iii) the formation channel that produces a wealth of binary systems hosting neutron stars or white dwarfs is not efficient in producing BSS.

We can also compare NGC 6266 with the sample of GCs searched for BSS with an approach similar to that used in this work. In particular, inspection of Tables 4 and 6 shows that in GCs hosting MSPs the specific frequency of BSS never exceeds 0.28. This sample includes the post-core collapsed globular cluster, NGC 6752, in which only a few BSS have been found (Sabbi et al. 2004). Conversely, up to now no MSPs have been found in M80 and NGC 288, the two clusters with the largest specific frequency of BSS measured so far (probably generated by different channels: primordial binaries in NGC 288 and collisional binaries in M80). The difficulty in modeling the observational biases involved in discovering radio pulsars in GCs prevents any firm conclusion to date, but an anticorrelation between MSP content and BSS specific frequency seems emerging. GCs with large populations of BSS seem to have a small population of MSP.

Financial support to this research has been provided by the Ministero dell'Istruzione, dell'Università e della Ricerca (MIUR) under the grant PRIN-2003023549. We also acknowledge the financial contribution from contract ASI-INAF I/023/05/0. We warmly thank M. Catelan for a careful reading of this paper and for helpful discussions. We also thank the referee for useful comments that 
improved the paper presentation.

\section{REFERENCES}

Bailyn, C. D., 1995, ARA\&A, 33, 133

Brocato, E., Buonanno, R., Malakhova, Y., \& Piersimoni, A. M. 1996, A\&A, 311, 778

Buonanno, R., Buscema, G., Corsi, C. E., Ferraro, I., \& Iannicola, G. 1983, A\&A, 126, 278

Buonanno, R. \& Iannicola, G. 1989, PASP, 101, 294

Caloi, V., 1987, "Conference on Faint Blue Stars, 2nd", Tucson, AZ, June 1-5, 1987, Proceedings, 539-542

Calzetti, D., de Marchi, G., Paresce, F., \& Shara, M. 1993, ApJ, 402, L1

Carretta, E. \& Gratton, R., 1997, A\&A, 121, 95

Catelan, M., Borissova, J., Sweigart, A. V., Spassova, N., 1998, ApJ, 494, 265

Cool, A. M., Grindlay, J. E., Cohn, H. N., Lugger, P. M., \& Bailyn, C. D. 1998, ApJ, 508, L75

Contreras, R., Catelan, M., Smith, Horace A., Pritzl, Barton J., Borissova, J., 2005, ApJL, 623, L117

D’Amico, N., Lyne, A. G., Manchester, R. N., Possenti, A. \& Camilo, F. 2001a, ApJ, 548, L171

D’Amico, N., Possenti, A., Manchester, R. N., Sarkissian, J., Lyne, A. G., \& Camilo, F. 2001b, in AIP Conf. Proc. 586, Relativistic Astrophysics: 20th Texas Symp., ed. J. C. Wheeler \& H. Martel (Melville: AIP), 526

Davies, M. B., Piotto, G., \& De Angeli, F. 2004, MNRAS, 349, 129

Djorgovski, S., \& King, I. R. 1986, ApJ, 305, L61

Djorgovski, S. 1993, in ASP Conf. Ser. 50, Structure and Dynamics of Globular Clusters, ed. S. G. Djorgovski \& G. Meylan (San Francisco: ASP), 373 (D93)

Djorgovski, S., \& Meylan, G. 1993, in ASP Conf. Ser. 50, Structure and Dynamics of Globular Clusters, ed. S. G. Djorgovski \& G. Meylan (San Francisco: ASP), 325 (DM93) 
Edmonds, P. D., Gilliland, R. L., Heinke, C. O., \& Grindlay, J. E. 2003, ApJ, 596, 1177

Ferraro, F. R., Fusi Pecci, F., \& Bellazzini, M. 1995, A\&A, 294, 80

Ferraro, F. R., et al. 1997, A\&A, 324, 915

Ferraro, F. R., Paltrinieri, B., Fusi Pecci, F., Rood, R. T., \& Dorman, B. 1998, ApJ, 500, 311

Ferraro, F. R., Paltrinieri, B., Rood, R. T., \& Dorman, B. 1999a, ApJ, 522, 983

Ferraro F. R., Messineo M., Fusi Pecci F., De Palo M. A., Straniero O., Chieffi A., Limongi M., 1999b, AJ, 118, 1738 (F99)

Ferraro, F. R., D’Amico, N., Possenti, A., Mignani, R. P., \& Paltrinieri, B. 2001, ApJ, 561, 337

Ferraro F. R., Sills A., Rood R. T., Paltrinieri B., Buonanno R., 2003a, ApJ, 588, 464 (F03)

Ferraro, F. R., Possenti, A., Sabbi, E., Lagani, P., Rood, R. T., D’Amico, N., \& Origlia, L. 2003b, ApJ, 595, 179

Ferraro F. R., Beccari G., Rood R. T., Bellazzini M., Sills A. \& Sabbi E., 2004, ApJ, 603, 127

Ferraro F. R., Sollima, A., Rood, R.T., Origlia, L., Pancino, E., Bellazzini, M., 2005, ApJ, in press, astro-ph/0510280

Gratton R. G., Bragaglia A., Carretta E., Clementini G., Desidera S., Grundahl F. \& Lucatello S., 2003, A\&A, 408, 529

Guhathakurta, P., Webster, Z. T., Yanny, B., Schneider, D. P., \& Bahcall, J. N. 1998, AJ, 116,1757

Harris, W. E. 1996, AJ, 112, 1487

Heinke, C. O., Edmonds, P. D., \& Grindlay, J. E. 2001, ApJ, 562, 363

Holtzman, J. A., Burrows, C. J., Casertano, S., Hester, J. J., Trauger, J. T., Watson, A. M., \& Worthey, G. 1995, PASP, 107, 1065

King, I. R. 1966, AJ, 71, 64

Kroupa, P. 2001, MNRAS, 322, 231 
Jacoby, B. A., Chandler, A. M., Backer, D. C., Anderson, S. B., \& Kulkarni, S. R. 2002, IAU Circ., 7783

Leonard, P. J. T. 1989, AJ, 98, 217

Lugger, P. M., Cohn, H., Grindlay, J. E. 1985, in IAU Symposium 113, Dynamics of Star Clusters, ed. J. Goodman and P.Hut (Dordrecht: Reidel), p.347

Mapelli, M., Sigurdsson, S., Colpi, M., Ferraro, F. R., Possenti, A., Rood, R. T., Sills, A. \& Beccari, G., 2004, ApJL, 605, 29

Meylan, G., \& Heggie, D. C. 1997, A\&A Rev., 8, 1

Montegriffo, P., Ferraro, F. R., Fusi Pecci, F., \& Origlia, L. 1995, MNRAS, 276, 739

Piotto, G., Zoccali, M., King, I. R., Djorgovski, S. G., Sosin, C., Rich, R. M., Meylan, G., 1999, ApJ, 118, 1727

Piotto, G.; King, I. R., Djorgovski, S. G., Sosin, C., Zoccali, M., Saviane, I., De Angeli, F., Riello, M., Recio-Blanco, A., Rich, R. M., Meylan, G., Renzini, A., 2002, A\&A, 391, 945

Piotto, G., et al. 2004, ApJ, 604, L109

Pooley, D., et al. 2003, ApJ, 591, L131

Possenti, A., D'Amico, N., Manchester, R. N., Camilo, F., Lyne, A. G., Sarkissian, J., \& Corongiu, A. 2003, ApJ, 599, 475

Rappaport, S., Pfahl, E., Rasio, F. A., \& Podsiadlowski, P. 2001, in Evolution of Binary and Multiple Star Systems, eds. P. Podsiadlowski, S. Rappaport, A. R. King, F. D'Antona, L. Burderi, ASP Conf. Ser. 229, 409

Renzini, A., \& Buzzoni, A. 1986, in Spectral Evolution of Galaxies, ed. C. Chiosi \& A. Renzini (Dordrecht: Reidel), 135

Rosenberg A., Piotto G., Saviane I. \& Aparicio A., 2000, A\&AS, 144, 5

Sabbi, E., Ferraro, F. R., Sills, A. \& Rood, R. T., 2004, ApJ, 617, 1296

Sandage, A. R. 1953, AJ, 58, 61

Sosin, C., et al. 1997, ApJ, 480, L35

Sigurdsson, S., \& Phinney, E. S. 1995, ApJS, 99, 609 
Stetson, P. B. 1994, PASP, 106, 250

Trager, S. C., King, I. R., \& Djorgovski, S. 1995, AJ, 109, 1912

Tsuchiya, T., Korchagin, V. I., Dinescu, D. I., 2004, MNRAS, 350, 1141

Verbunt, F., 2003 in New Horizons in Globular Cluster Astronomy, ASP Conf. Ser. 296, 245 
Table 1. Surface density in different annuli around the NGC 6266 center.

\begin{tabular}{cccc|cccc}
\hline \hline & & & & & & & \\
$r 1$ & $r 2$ & $\frac{N}{\operatorname{arcsec}^{2}}$ & err & $r 1$ & $r 2$ & $\frac{N}{\operatorname{arcsec}^{2}}$ & err \\
\hline 0.0 & 2.0 & 10.89 & 0.04 & 371.0 & 404.0 & 0.034 & 0.026 \\
2.0 & 4.0 & 10.49 & 0.04 & 404.0 & 437.0 & 0.032 & 0.025 \\
4.0 & 6.0 & 9.80 & 0.02 & 437.0 & 470.0 & 0.032 & 0.020 \\
6.0 & 8.0 & 9.07 & 0.03 & 470.0 & 514.5 & 0.027 & 0.025 \\
8.0 & 10.0 & 8.28 & 0.04 & 514.5 & 559.0 & 0.027 & 0.036 \\
10.0 & 12.0 & 6.69 & 0.04 & 559.0 & 603.5 & 0.028 & 0.029 \\
12.0 & 14.0 & 5.93 & 0.05 & 603.5 & 648.0 & 0.028 & 0.023 \\
23.0 & 33.0 & 2.03 & 0.05 & 648.0 & 692.5 & 0.027 & 0.026 \\
33.0 & 43.0 & 1.54 & 0.03 & 692.5 & 737.0 & 0.027 & 0.024 \\
43.0 & 53.0 & 1.13 & 0.03 & 737.0 & 791.0 & 0.024 & 0.032 \\
53.0 & 63.0 & 0.869 & 0.025 & 791.0 & 845.0 & 0.024 & 0.004 \\
63.0 & 73.0 & 0.647 & 0.025 & 845.0 & 899.0 & 0.026 & 0.003 \\
73.0 & 83.0 & 0.524 & 0.012 & 899.0 & 953.0 & 0.026 & 0.021 \\
83.0 & 93.0 & 0.415 & 0.021 & 953.0 & 1007.0 & 0.025 & 0.021 \\
140.0 & 173.0 & 0.107 & 0.003 & 1007.0 & 1061.0 & 0.025 & 0.024 \\
173.0 & 206.0 & 0.081 & 0.037 & 1061.0 & 1116.0 & 0.027 & 0.009 \\
206.0 & 239.0 & 0.061 & 0.042 & 1116.0 & 1171.0 & 0.027 & 0.008 \\
239.0 & 272.0 & 0.045 & 0.046 & 1171.0 & 1226.0 & 0.029 & 0.015 \\
272.0 & 305.0 & 0.038 & 0.003 & 1226.0 & 1281.0 & 0.029 & 0.008 \\
305.0 & 338.0 & 0.037 & 0.002 & 1281.0 & 1486.0 & 0.024 & 0.101 \\
338.0 & 371.0 & 0.035 & 0.022 & & & & \\
\hline \hline
\end{tabular}


Table 2. NGC 6266 parameters

\begin{tabular}{cccccccc}
\hline \hline & $\mathrm{E}(\mathrm{B}-\mathrm{V})$ & $(m-M)_{0}$ & $\mu_{v}(0)$ & $\mu_{0, v}$ & $\begin{array}{c}r_{c} \\
{[\operatorname{arcsec}]}\end{array}$ & $\mathrm{c}$ & $\begin{array}{c}\log \left(\rho_{0}\right) \\
{\left[M_{\odot} \mathrm{pc}^{-3}\right]}\end{array}$ \\
\hline DM93 & 0.50 & 13.70 & 15.2 & 13.64 & 10.7 & 1.8 & 5.7 \\
This Paper & 0.47 & 14.11 & 15.2 & 13.74 & 19 & 1.5 & 5.46 \\
\hline \hline
\end{tabular}

Note. - $E(B-V)$ from Harris 1996

Table 3. Structural paramenters for a sample of clusters searched for BSS

\begin{tabular}{cccccccc}
\hline \hline Cluster & {$[\mathrm{Fe} / \mathrm{H}]$} & $\mathrm{E}(\mathrm{B}-\mathrm{V})$ & $(M-m)_{0}$ & $\begin{array}{c}\text { Dist } \\
{[\mathrm{Kpc}]}\end{array}$ & $\begin{array}{c}r_{c} \\
{[\operatorname{arcsec}]}\end{array}$ & $\begin{array}{c}\log \left(\rho_{o}\right) \\
{\left[M_{\odot} \mathrm{pc}^{-3}\right]}\end{array}$ \\
& & & & & & \multicolumn{2}{c}{ This Paper $^{\text {DM93 }}$} \\
\hline M 3 & -1.34 & 0.01 & 15.03 & 10.1 & 30 & 4.0 & 3.5 \\
M 13 & -1.39 & 0.02 & 14.43 & 7.7 & 40 & 3.9 & 3.4 \\
M 80 & -1.41 & 0.18 & 14.96 & 9.8 & 6.5 & 5.4 & 5.4 \\
M 10 & -1.41 & 0.28 & 13.38 & 4.7 & 40 & 4.0 & 3.8 \\
NGC 288 & -1.07 & 0.03 & 14.73 & 8.8 & 85 & 2.3 & 2.1 \\
M 92 & -2.16 & 0.02 & 14.78 & 9.04 & 14 & 4.7 & 4.4 \\
NGC 6266 & -1.07 & 0.47 & 14.11 & 6.6 & 19 & 5.5 & 5.7 \\
NGC 6752* & -1.42 & 0.04 & 13.18 & 4.3 & 5.7 & 5.6 & 5.2 \\
NGC 104 & -0.70 & 0.04 & 13.32 & 4.6 & 21 & 5.3 & 5.1 \\
\hline \hline
\end{tabular}

Note. — * Post core collapse cluster. 
Table 4. BSS population in a sample of GGCs.

\begin{tabular}{ccccc}
\hline \hline & & & & \\
Cluster & $N_{\mathrm{BSS}}$ & $N_{\mathrm{HB}}$ & $F_{\mathrm{HB}}^{\mathrm{BSS}}$ & $r_{1 / 2}^{\mathrm{BSS}} / r_{c}$ \\
& & & & \\
\hline M13 & 16 & 228 & 0.07 & 1.15 \\
NGC 6266 & 47 & 395 & 0.12 & 0.63 \\
NGC 104† & 53 & 314 & 0.17 & 0.87 \\
NGC 6752* & 28 & 156 & 0.18 & 0.82 \\
M10 & 22 & 81 & 0.27 & 0.85 \\
M3 & 72 & 257 & 0.28 & 0.73 \\
M92 & 53 & 160 & 0.33 & 1.07 \\
M80 & 129 & 293 & 0.44 & 1.07 \\
NGC 288 & 24 & 26 & 0.92 & 0.71 \\
\hline \hline
\end{tabular}

Note. - $†$ In 47 Tuc the selection of BSS and HB has been performed using the CMD plane $m_{\mathrm{F} 218 \mathrm{~W}}, m_{\mathrm{F} 218 \mathrm{~W}}-m_{\mathrm{F} 439 \mathrm{~W}}$ instead of the $m_{\mathrm{F} 255 \mathrm{~W}}, m_{\mathrm{F} 255 \mathrm{~W}}-m_{\mathrm{F} 336 \mathrm{~W}}$ plane.

Note. — * Post core collapse cluster. 
Table 5. Expected number of single star $\left(N_{s s}\right)$ and binary-binary $\left(N_{b b}\right)$ encounters per Gyr

\begin{tabular}{ccccc}
\hline \hline Cluster & $a_{h s}(A U)$ & $\begin{array}{c}\sigma_{0} \\
{\left[\mathrm{~km} \mathrm{~s}^{-1}\right]}\end{array}$ & $N_{s s}$ & $N_{b b}$ \\
\hline M13 & 0.39 & 7.1 & 0.07 & 0.17 \\
NGC 6266 & 0.10 & 14.3 & 3.19 & 2.07 \\
NGC 104 & 0.15 & 11.5 & 1 & 1 \\
NGC 6752 & 0.98 & 4.5 & 0.16 & 1.04 \\
M10 & 0.45 & 6.6 & 0.05 & 0.11 \\
M3 & 0.63 & 5.6 & 0.16 & 0.70 \\
M92 & 0.57 & 5.9 & 0.28 & 1.04 \\
M80 & 0.13 & 12.4 & 0.33 & 0.29 \\
NGC 288 & 2.36 & 2.9 & 0 & 0.02 \\
\hline \hline
\end{tabular}

Table 6. MSP content, destruction and formation rate

\begin{tabular}{cccccc}
\hline \hline & Isolated & Binary & & & \\
Cluster & PSRs & PSRs & $\mathcal{R}_{\text {form }}$ & $\mathcal{R}_{\text {dist }}$ & $\mathcal{R}_{\text {form }} / \mathcal{R}_{\text {dist }}$ \\
& & & & & \\
\hline M13 & 2 & 3 & $6.51 \times 10^{-2}$ & $7.18 \times 10^{-2}$ & 1.1 \\
NGC 6266 & 0 & 6 & 2.65 & 1.09 & 2.97 \\
NGC 104 & 7 & 15 & 1.00 & 1.00 & 1.00 \\
NGC 6752 & 4 & 1 & 0.18 & 6.72 & $3.21 \times 10^{-2}$ \\
M10 & 0 & 0 & $4.61 \times 10^{-2}$ & 0.14 & 0.40 \\
M3 & 0 & $4 \dagger$ & 0.11 & $8.83 \times 10^{-2}$ & 1.58 \\
M92 & 0 & 0 & 0.21 & 0.47 & 0.56 \\
M80 & 0 & 0 & 0.51 & 1.97 & 0.32 \\
NGC288 & 0 & 0 & $1.6 \times 10^{-3}$ & $4.72 \times 10^{-3}$ & 0.41 \\
\hline \hline
\end{tabular}

Note. - $†$ For 3 of these pulsars no timing/orbital solution has been published yet. 


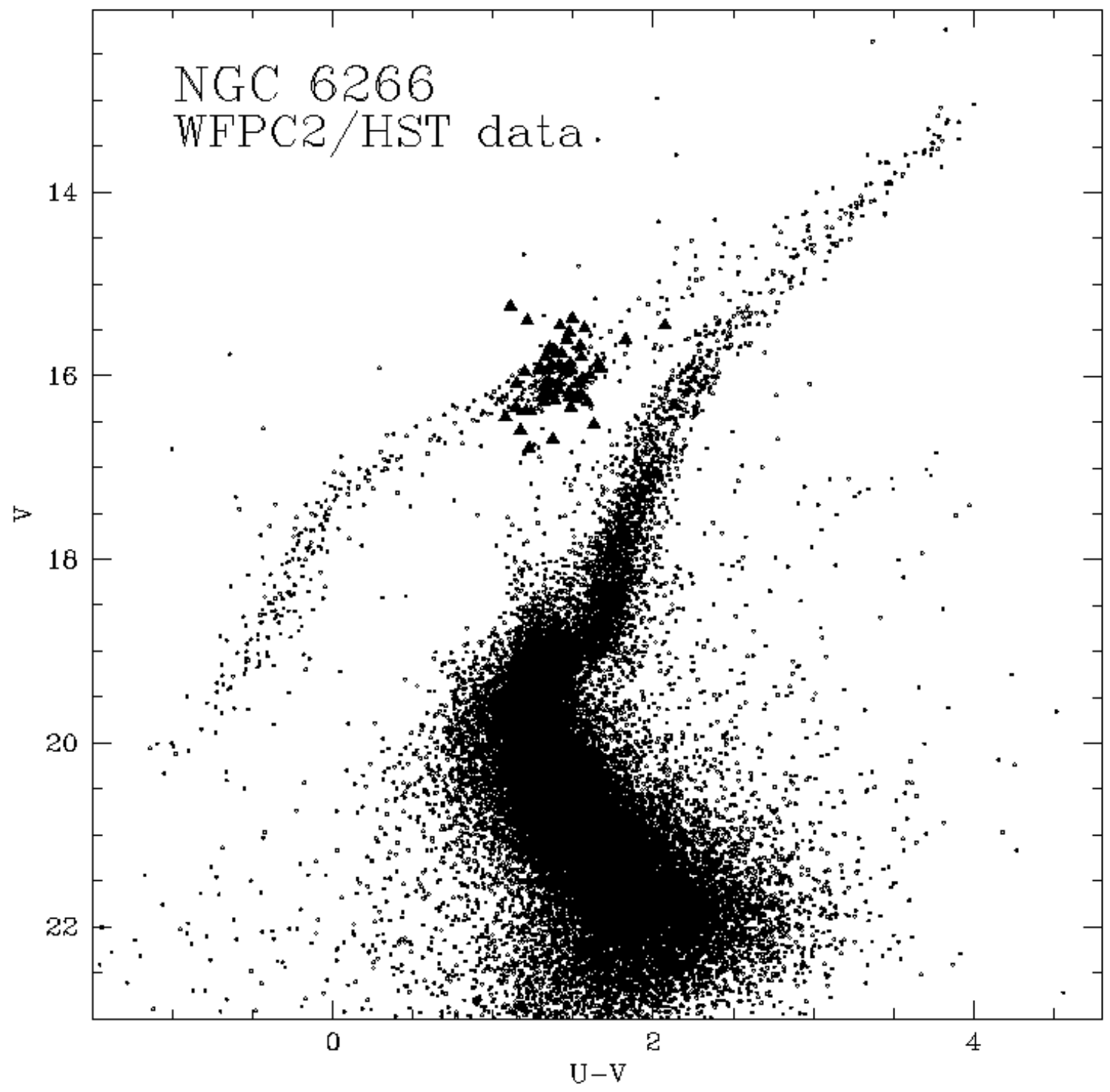

Fig. 1. - CMDs for stars in the HST/WFPC2 FoV. RRLyrae stars lying in HST/WFPC2 field of view are marked with large filled triangles. 


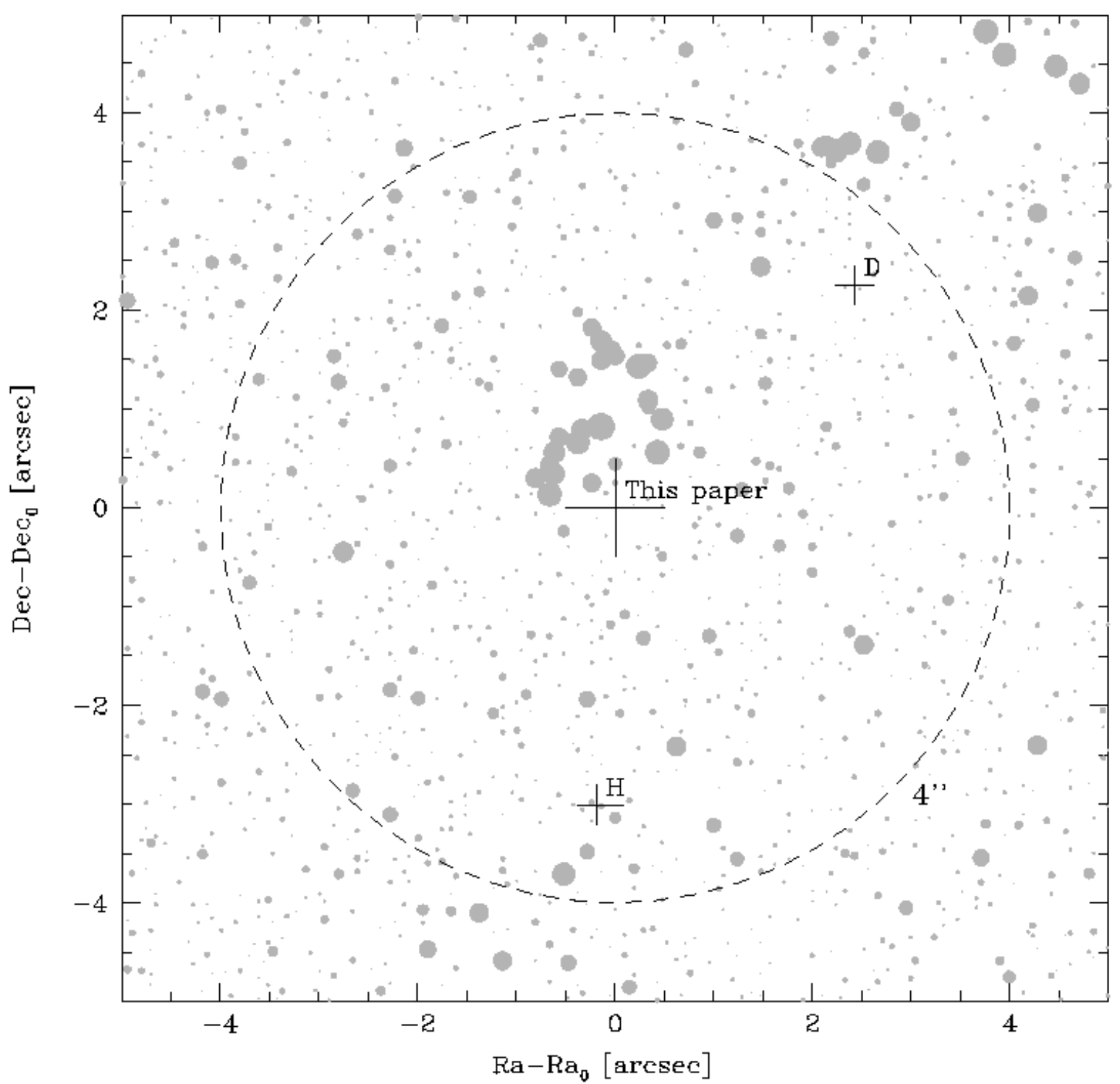

Fig. 2.- Computer map of the inner $10^{\prime \prime} \times 10^{\prime \prime}$ region of the cluster. The large cross at $(0,0)$ coordinates indicates the adopted $C_{\text {grav }}$. The $C_{\text {lum }}$ by Djorgovski (1993) and Harris (1996) are labeled with D and H, respectively. 


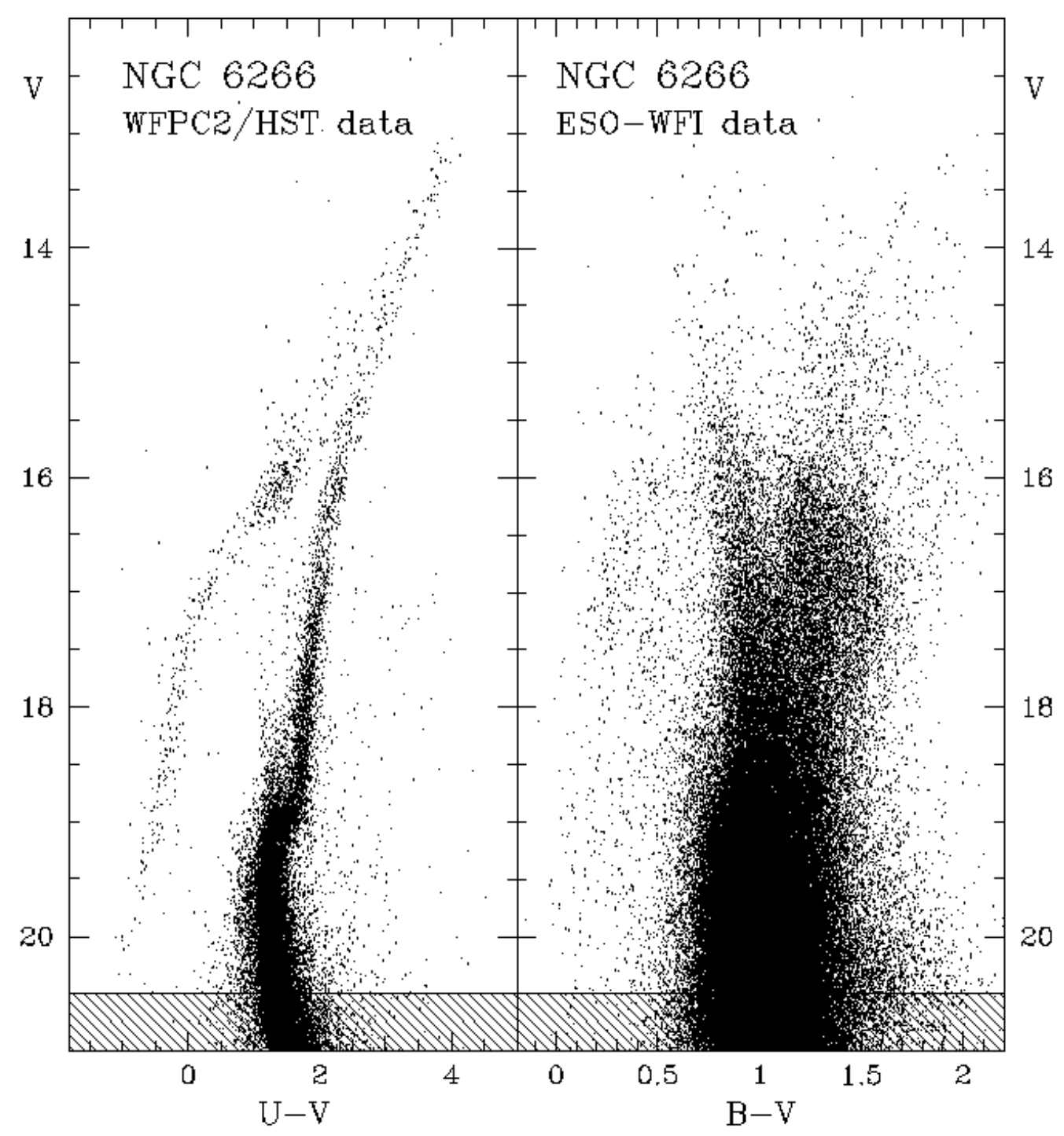

Fig. 3.- CMDs for stars in the two samples. Left panel: the high resolution HST catalog $\left(r<93^{\prime \prime}\right)$ in the (F555W, F336W - F555W) plane. Right panel: the wide-field WFI catalog in the $(V, B-V)$ plane, only stars with $r>140^{\prime \prime}$ from the cluster center are plotted. Stars with $V>20.5$ (shaded region) have been excluded from the construction of the density profile. 


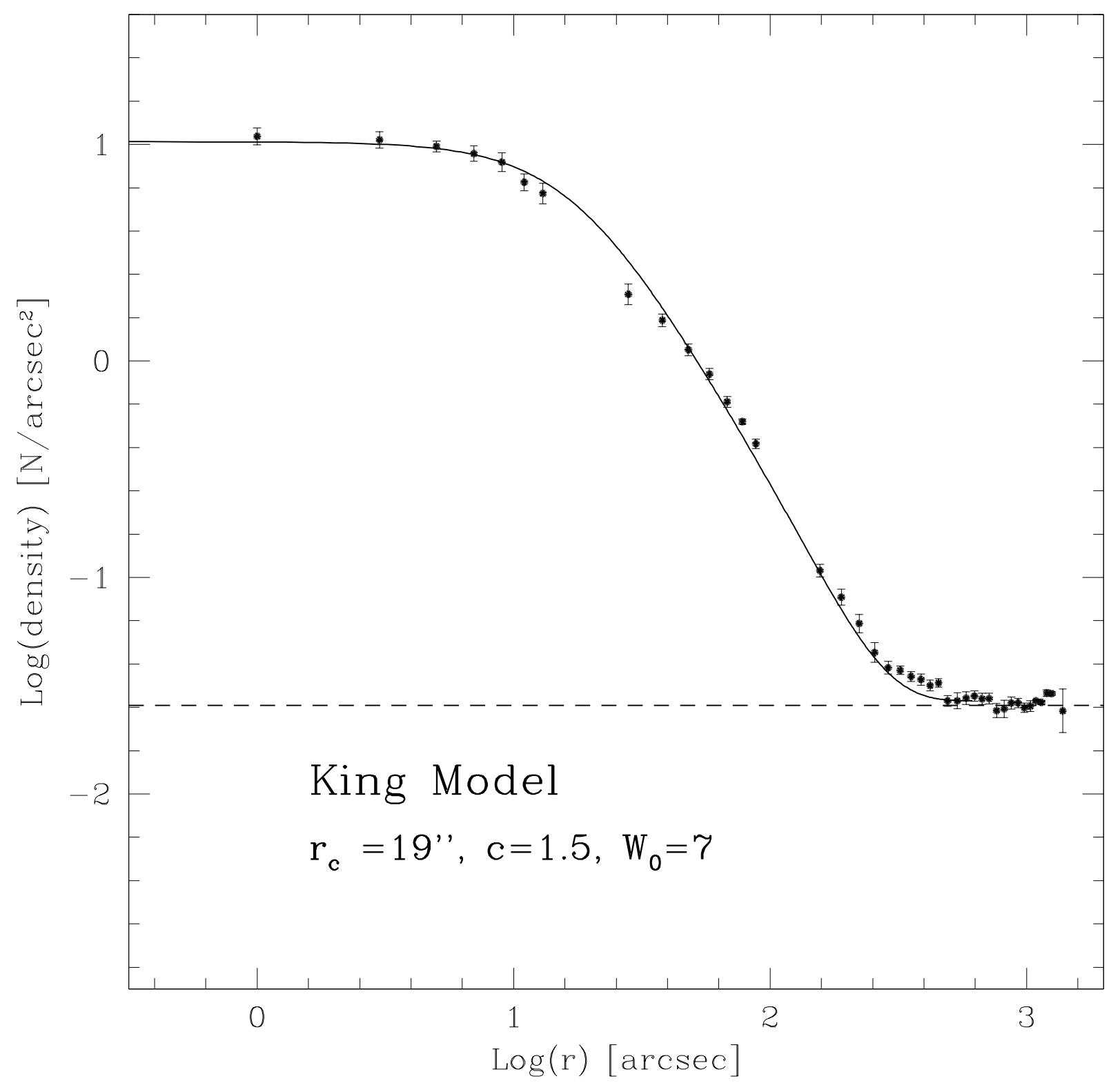

Fig. 4.- Observed radial density profile with respect to the the adopted $C_{\text {grav }}$. The solid line is the best fit King model $(c=1.5)$ to the observed density profile over the entire extension. A value of $\sim 92 \mathrm{stars} \mathrm{arcmin}^{-2}$ is representative field contamination (dashed horizontal line). The adopted value for the parameter $W_{0}$ (the central potential parameter as defined by King 1996) is also reported. 


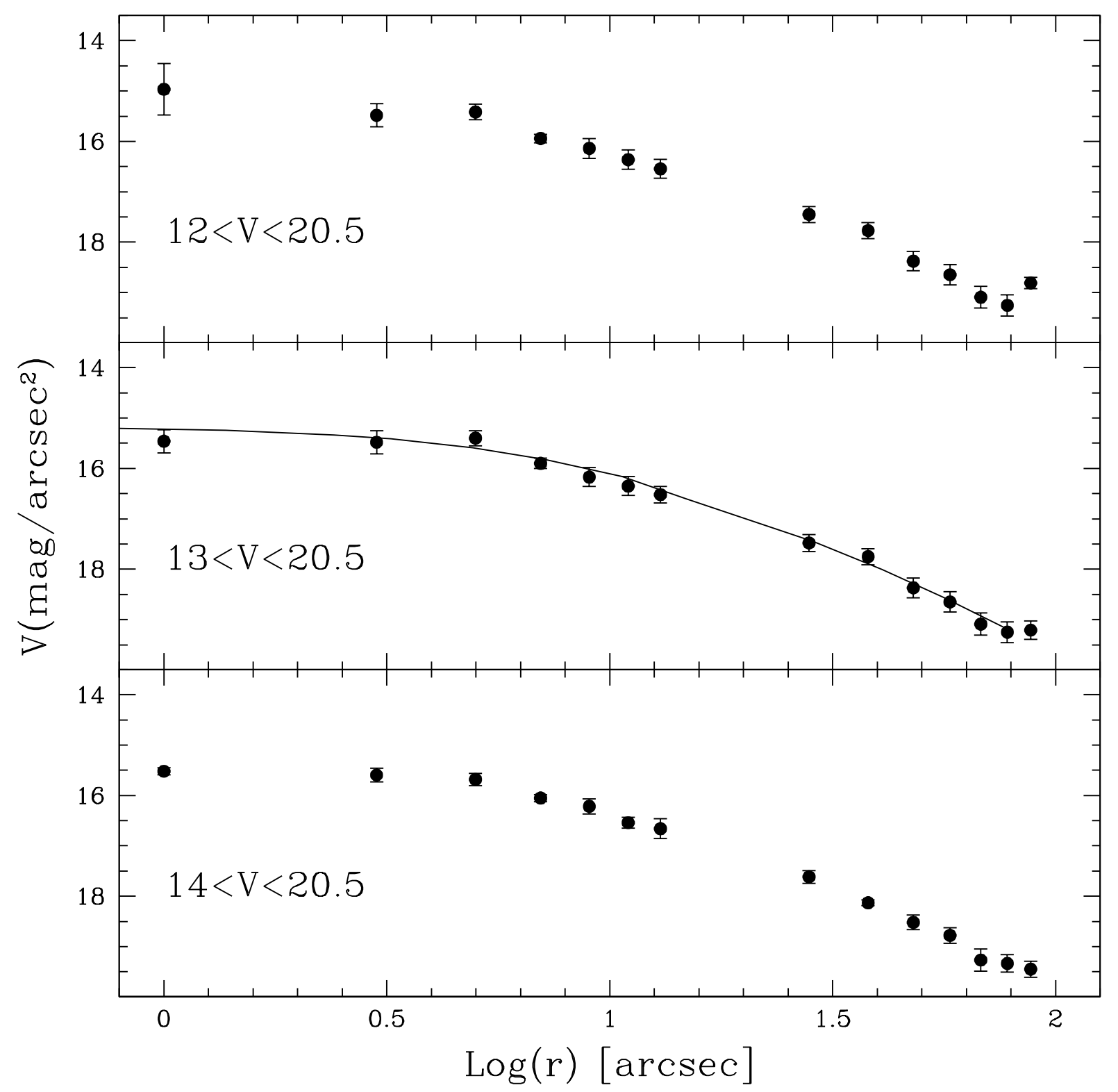

Fig. 5.- Radial brightness profiles computed, for the WFPC2/HST sample, by removing the stars brighter than $V=12,13,14$, respectively, the faintest limit is $V=20.5$ (see Figure 3 ). The solid line is the best-fit solution. 

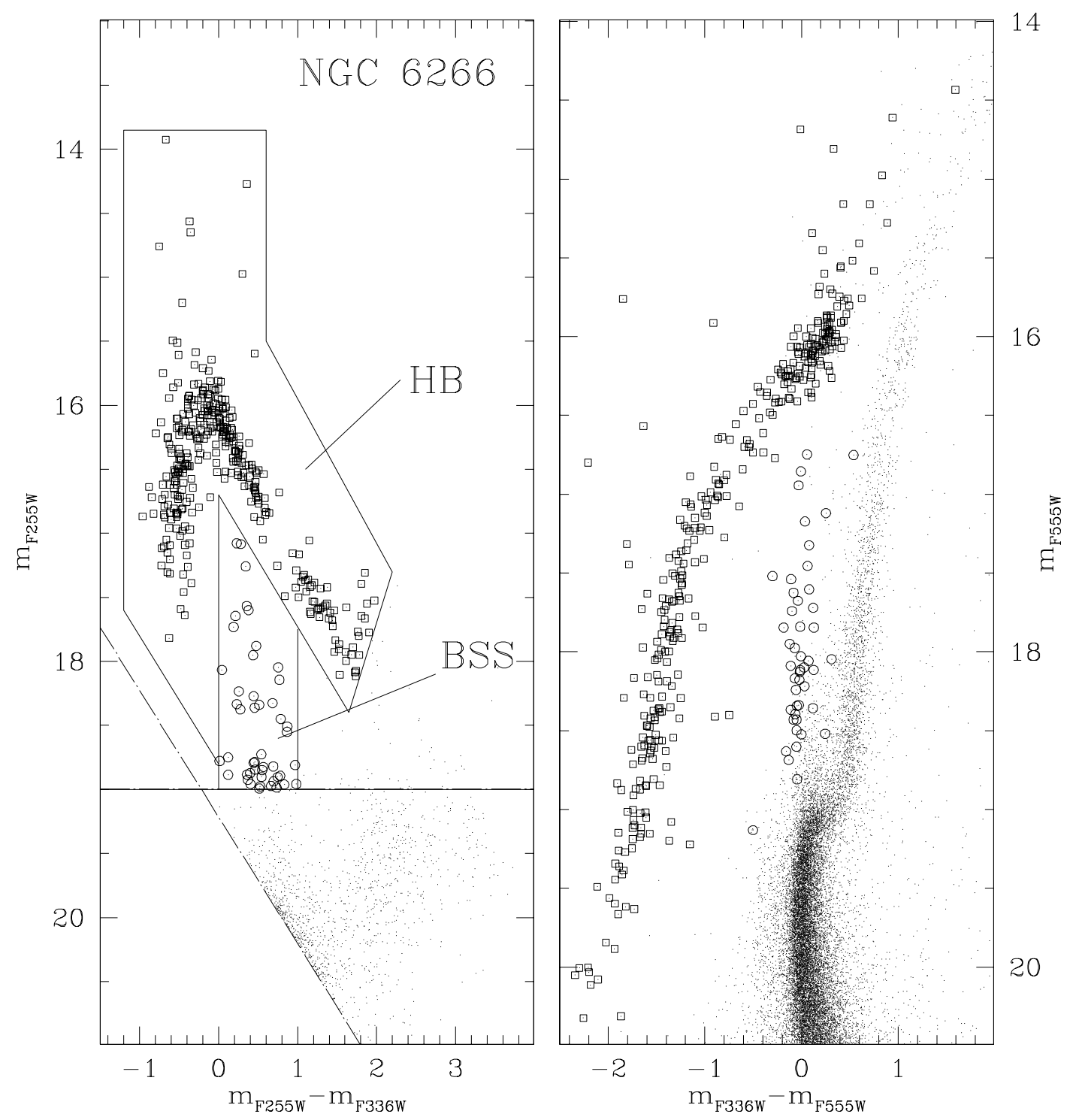

Fig. 6. - BSS (open circles) and HB (open squares) in the (F255W, F255W - F336W) CMD of NGC 6266 (left panel) and (F555W, F336W - F555W) CMD (right panel). Note that only non variable HB stars are plotted. The adopted selection boxes in the UV-CMD are shown. 


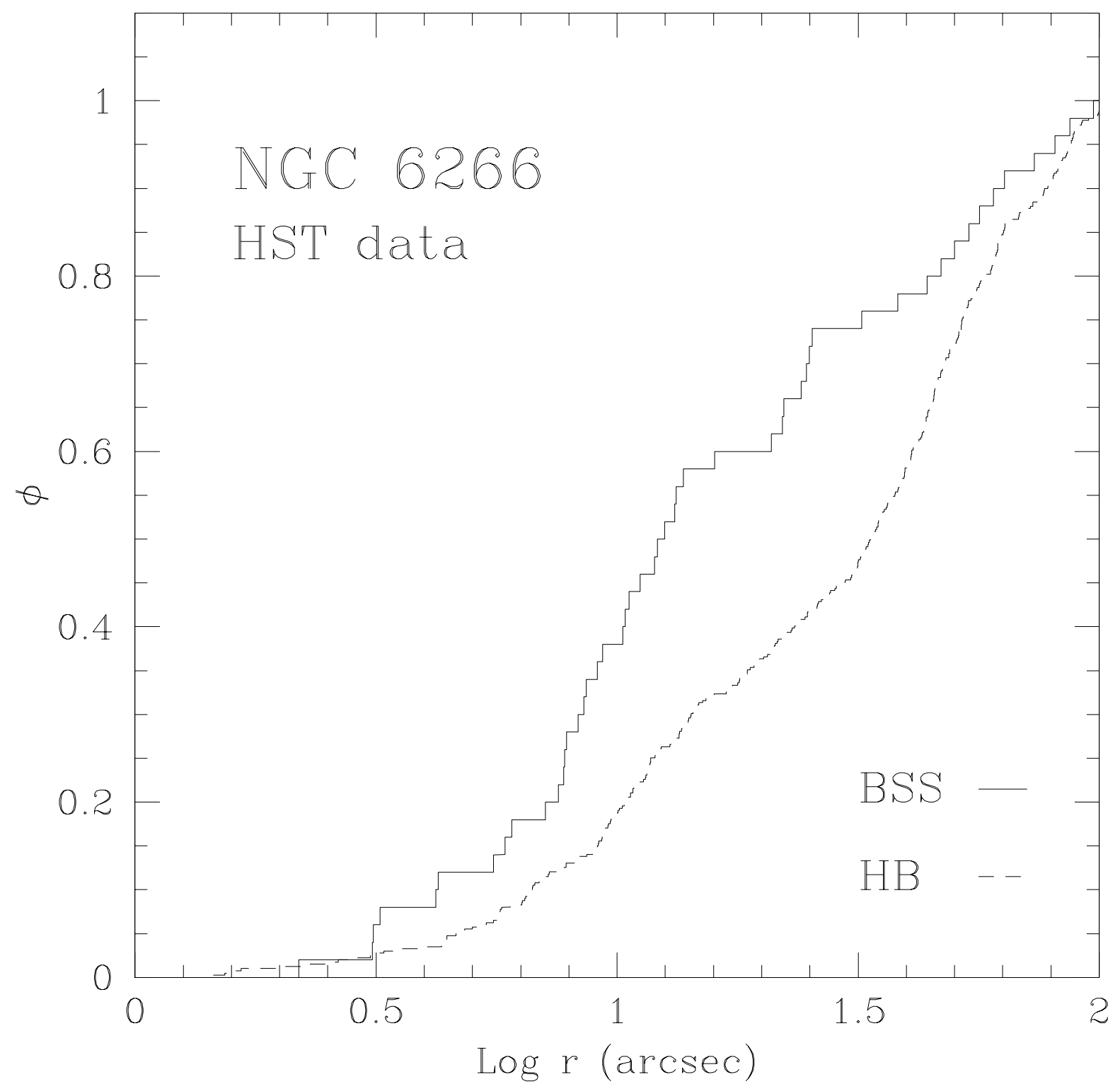

Fig. 7.- Cumulative radial distribution of BSS (solid line) with respect to the HB stars (dashed line) as a function of their projected distance $(r)$ from the cluster center. The probability that the two populations are extracted from the same distribution is $P=2 \times 10^{-4}$ 


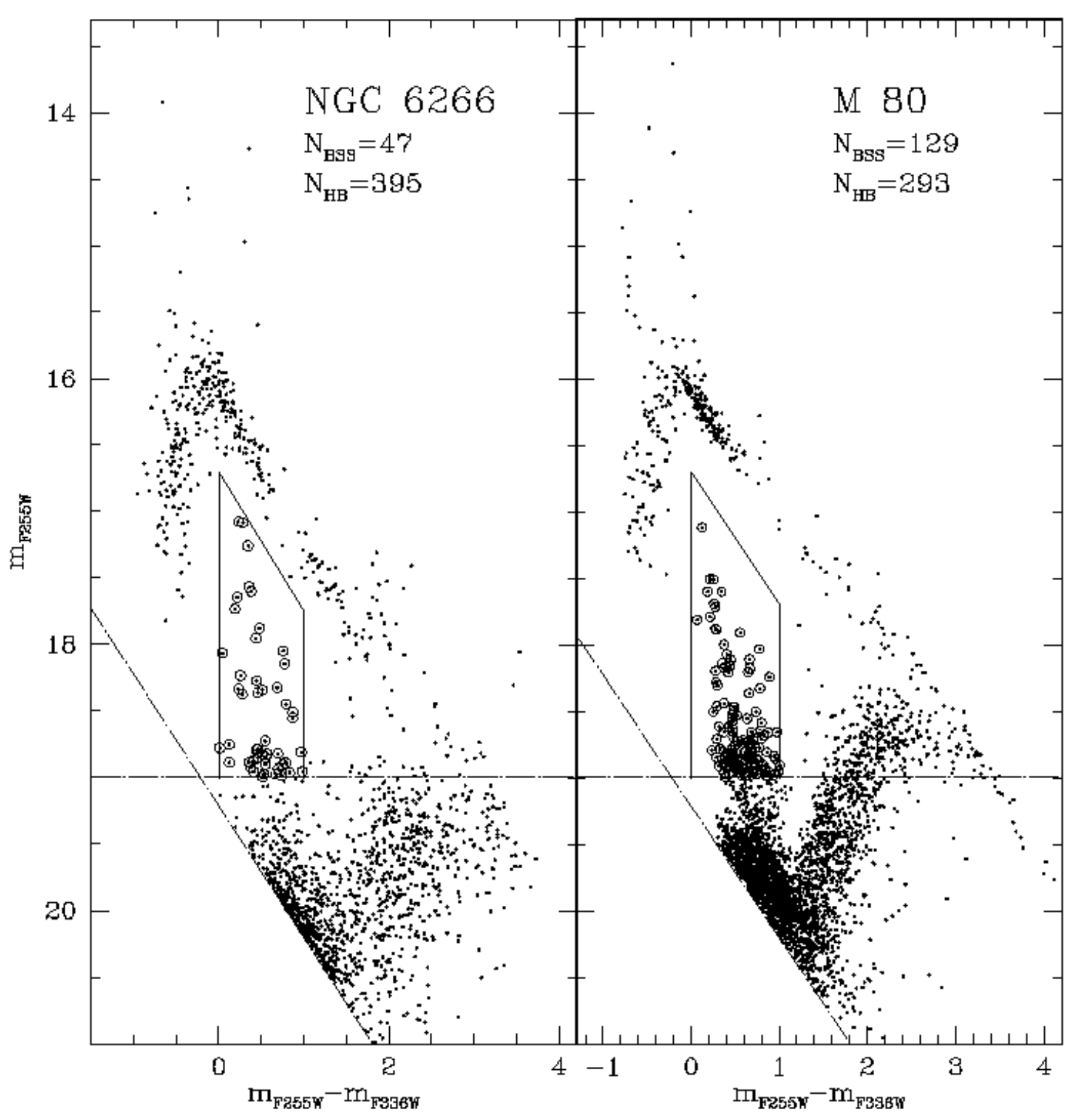

Fig. 8. - BSS (open circles) in the (F255W, F255W - F336W) CMDs of NGC 6266 (left panel) and M80 (right panel) for comparison. The CMDs have been shifted as suggested in F03. The horizontal line shows the limiting magnitude of the adopted selection. 